\title{
Butein sensitizes HeLa cells to cisplatin through the AKT and ERK/p38 MAPK pathways by targeting FoxO3a
}

\author{
LIRUI ZHANG ${ }^{1}$, XIAOFENG YANG ${ }^{1}, \mathrm{XU} \mathrm{LI}^{2}, \mathrm{CHEN} \mathrm{LI}^{1}, \mathrm{LE} \mathrm{ZHAO}^{2}, \mathrm{YUANYUAN}_{\mathrm{ZHOU}}^{2}$ and HUILIAN HOU ${ }^{3}$ \\ ${ }^{1}$ Department of Gynecology and Obstetrics, ${ }^{2}$ Center for Translational Medicine and ${ }^{3}$ Department of Pathology, \\ The First Affiliated Hospital of Xi'an Jiaotong University School of Medicine, Xi'an, Shaanxi 710061, P.R. China
}

Received March 13, 2015; Accepted August 6, 2015

DOI: 10.3892/ijmm.2015.2324

\begin{abstract}
Drug resistance remains a major challenge in cancer therapy. Butein, a polyphenolic compound, has been shown to exhibit anticancer activity through the inhibition of the activation of the protein kinase $\mathrm{B}$ (PKB/AKT) and mitogen-activated protein kinase (MAPK) pathways, which are two pathways known to be involved in resistance to cisplatin. Hence, we hypotheiszed that butein may be a chemosensitizer to cisplatin. In the present study, we demonstrated that butein synergistically enhanced the growth inhibitory and apoptosis-inducing effects of cisplatin on HeLa cells. Moreover, the combination of butein and cisplatin led to G1 phase arrest. We then aimed to explore the underlying mechanisms. We found that butein inhibited the activation of AKT, extracellular signal-regulated kinase (ERKs) and p38 kinases in the presence of cisplatin. The use of the AKT inhibitor, LY294002, in combination with cisplatin, induced an increase in apoptosis compared to treatment with cisplatin alone, although this effect was not as prominent as that exerted by butein in combination with cisplatin. Of note, the inhibition of ERK or p38 MAPK by U0126 or SB203580, respectively, decreased the apoptosis induced by cisplatin; however, enhanced apoptotic effects were observed with the use of ERK/p38 MAPK inhibitor in combination with butein. These data suggest that the AKT and ERK/p38 MAPK pathways are involved in the synergistic effects of butein and cisplatin. Furthermore, co-treatment with butein and cisplatin promoted the nuclear translocation and expression of forkhead box O3a (FoxO3 or FoxO3a). FoxO3a may be the key molecule on which these pathways converge and is thus implicated in the synergistic effects of butein and cisplatin. This was further confirmed by the RNAi-mediated suppression of FoxO3a. FoxO3a target genes involved in cell cycle progression and apoptosis were also investigated, and combined treatment with butein and cisplatin resulted in the
\end{abstract}

Correspondence to: Dr Lirui Zhang, Department of Gynecology and Obstetrics, The First Affiliated Hospital of Xi'an Jiaotong University School of Medicine, 277 Yanta Road, Xi'an, Shaanxi 710061, P.R. China

E-mail: lirui_zhang@163.com

Key words: butein, cisplatin, protein kinase B, extracellular signalregulated kinases, p38 kinases, forkhead box O3 downregulation of cyclin D1 and Bcl-2 and the upregulation of p27 and Bax. In addition, the combination of both agents markedly inhibited tumor growth and increased the expression of FoxO3a in mouse tumor xenograft models of cervical cancer. Taken together, to the best of our knowledge, our results reveal for the first time that butein sensitizes cervical cancer cells to cisplatin in vitro and in vivo, and these effects of butien may be related to the inhibition of the activation of the AKT and ERK/ p38 MAPK pathways by targeting FoxO3a.

\section{Introduction}

Cisplatin (cis-diamminedichloroplatinum II, CDDP) is one of the most effective chemotherapeutic agents used in the treatment of advanced cervical cancer. However, chemoresistance is the major reason of treatment failure. Therefore, in order to improve the clinical outcome, more effective and tolerable combination treatment strategies are required (1).

DNA damage induced by cisplatin triggers cell cycle arrest and this then leads to apoptosis. Nevertheless, it has been demonstrated that various survival signals, including mitogen-activated protein kinase (MAPK) and phosphoinositide 3-kinase (PI3K)/protein kinase B (PKB/AKT) signaling, are activated by cisplatin treatment and may thus be responsible for the chemoresistance (2). There are 3 major subfamilies of the MAPK family: the extracellular signal-regulated kinases (ERKs), the c-Jun, N-terminal kinases (JNKs) and the p38 kinases, regulating a variety of physiological processes such as cell growth, metabolism, differentiation and cell death; however, MAPK signal dysfunction could results in tumorigenesis and drug resistance. PKB/AKT, a serine/ threonine kinase, functions as an oncogene and has also been implicated in resistance to chemotherapy drugs. It has been reported that the inhibition of AKT enhances the therapeutic activity of paclitaxel against cervical carcinomas $(3,4)$.

3,4,20,40-Tetrahydroxychalcone (butein), as a polyphenolic compound, is used as a food additive and a traditional herbal medicine to alleviate pain, and in the treatment of parasitic and thrombotic diseases (5). Previous studies, including ours have demonstrated that butein exerts anticancer activity, and suppresses the proliferation of a number of human cancers, including breast carcinoma, colon caner, hepatocellular carcinoma and bladder cancer (6-10). The anticancer activity of butein has been reported to involve the regulation of AKT/ 
MAPK signaling. Butein has been shown to inhibit the activation of ERK, JNK and p38 in human hepatocellular carcinoma and breast cancer cells $(9,11)$. Moreover, butein has been shown to inhibit AKT phosphorylation, resulting in the suppression of in breast cancer and prostate cancer cell growth $(7,12)$. Based on the above-mentioned facts, we hypothesized that butein may sensitize cervical cancer cells to cisplatin by suppressing the activation of the MAPK and PI3K/AKT signaling pathways.

\section{Materials and methods}

Drugs and antibodies. Butein and cisplatin (CDDP) were obtained from Sigma (St. Louis, MO, USA), and were dissolved in dimethyl sulfoxide (DMSO) and stored at $-20^{\circ} \mathrm{C}$ until use. The ERK inhibitor, U0126, p38 inhibitor, SB203580, and the AKT inhibitor, LY294002, were obtained from Sigma and used at final concentrations of 10,20 and $20 \mu \mathrm{M}$, respectively. The following antibodies were used in western blot analysis: antiERK1/2 (\#9102; 1:1,000), anti-p-ERK1/2 (Thr202/Tyr204) (\#9101; 1:1,000), anti-p38 (\#9212; 1:1,000), anti-p-p38 (Thr180/ Tyr182) (\#9211; 1:1,000), anti-Akt (\#9272; 1:1,000) and antip-Akt (Ser413) (\#9271; 1:1,000) (all purchased from Cell Signaling Technology, Danvers, MA, USA). $\beta$-actin (sc-4778; 1:5,000), forkhead box O3a (FoxO3 or FoxO3a; sc-9812; 1:1,000), Bax (sc-7480; 1:1,000), Bcl-2 (sc-7382; 1:1,000), p27 (sc-1641; 1:1,000) and cyclin D1 (sc-718; 1:1,000) antibodies were purchased from Santa Cruz Biotechnology (Santa Cruz, CA, USA). Horseradish peroxidase (HRP)-conjugated goat anti-mouse/anti-rabbit immunoglobulin (IgG; ab6721; 1:1,000) was obtained from Abcam (Hong Kong, China).

Cell culture. HeLa human cervical carcinoma cells were obtained from the Shanghai Cell Bank of Chinese Academy of Sciences (Shanghai, China). The cells were cultured in RPMI-1640 medium (Gibco-BRL, Grand Island, NY, USA) supplemented with $10 \%$ bovine calf serum, $100 \mathrm{U} / \mathrm{ml}$ penicillin and $100 \mathrm{mg} / \mathrm{ml}$ streptomycin, and maintained at $37^{\circ} \mathrm{C}$ in a humidified atmosphere of $5 \% \mathrm{CO}_{2}$.

Cell viability assay. The viability of the HeLa cells following treatment with butein and cisplatin, alone or in combination, was determined using the methylthiazol tetrazolium (MTT) assay. The Cells were digested and diluted to $1 \times 10^{5} / \mathrm{ml}$. Subsequently, $200 \mu \mathrm{l}$ of the single cell suspension were seeded into 96 -well culture plates. Following overnight incubation, the cells were washed with phosphate-buffered saline (PBS) and divided into groups [control (untreated), butein-treated, cisplatin-treated and butein + cisplatin-treated cells]; each group had 6-wells in a single line. The groups were placed in starvation medium with $0.2 \%$ DMSO or the drugs and incubated for a further $48 \mathrm{~h}$. The drug-containing medium was then replaced with fresh medium. MTT solution $(500 \mathrm{mg} / \mathrm{ml})$ was added to the medium and this was maintained at $37^{\circ} \mathrm{C}$ for $4 \mathrm{~h}$. The cells were cultured at $37^{\circ} \mathrm{C}$ for $4 \mathrm{~h}, 150 \mu \mathrm{l}$ DMSO was added, and the $570 \mathrm{~nm}$ wavelength absorption values were measured using an EnSpire Multimode plate reader (Perkin-Elmer, Waltham, MA, USA). All experiments were performed in triplicate, and repeated 3 times. Cell viability and growth inhibition were calculated as follows: cell viability rate $=$ A570 value of the drug treated group/A570 value of the control untreated group x100; growth inhibition rate $=1$ - cell viability rate. The interaction between the 2 drugs was judged according to a method described in the study by Jin (13). Briefly, a q-value was calculated according to the formula: $\mathrm{q}=\mathrm{Ea}+\mathrm{b} /(\mathrm{Ea}+\mathrm{Eb}-\mathrm{Ea} \times \mathrm{Eb})$. The 2 drugs were considered to have additive effects if $0.85<\mathrm{q}<1.15$, or synergistic effects if $\mathrm{q}>1.15$, and are antagonistic if $\mathrm{q}<0.85$.

Apoptosis assay. Apoptosis was assessed using the Annexin V-FITC apoptosis detection kit according to the manufacturer's instructions (Sigma-Aldrich, Oakville, ON, Canada). Approximately $10^{6}$ cells were seeded onto sterile flat-bottom $25-\mathrm{cm}^{2}$ culture flasks. The cells were treated with cisplatin and butein according to the experimental design. Following incubation for $48 \mathrm{~h}$, the cells were collected, washed in PBS and resuspended in $500 \mathrm{ml}$ of $1 \mathrm{X}$ Annexin $\mathrm{V}$ binding buffer and then incubated at room temperature with teh Annexin V-FITC and PI stain in the absence of light. Following a 10-min incubation, the cells were immediately analyzed by flow cytometry. Annexin V staining was detected as green fluorescence and PI as red fluorescence. The percentage of cells undergoing apoptosis was determined by 3 independent experiments.

Cell cycle analysis. Cells were seeded at a density of $2 \times 10^{5}$ cells/ well in 6-well plates. Following overnight incubation, the cells were then exposed to butein and/or cisplatin for $48 \mathrm{~h}$. Following incubation, the cells were then fixed for $1 \mathrm{~h}$ in icecold $70 \%$ ethanol and incubated for $30 \mathrm{~min}$ at $37^{\circ} \mathrm{C}$ with $0.5 \mathrm{U}$ of RNase A (Sigma-Aldrich). DNA was then stained for $10 \mathrm{~min}$ with $50 \mu \mathrm{g} / \mathrm{ml}$ of PI and the cells analyzed using a flow cytometer (FACSCalibur; BD Biosciences, San Jose, CA, USA).

Western blot analysis. The cells were lysed with RIPA buffer (150 mM NaCl, 1.0\% Nonidet P-40, 0.5\% sodium deoxycholate, $0.1 \%$ SDS, $50 \mathrm{mM}$ Tris, $\mathrm{pH}$ 8.0) containing protease inhibitor cocktail (Roche Applied Science, Mannheim, Germany). Following centrifugation for $10 \mathrm{~min}$ at $10000 \mathrm{x} \mathrm{g}$ at $4^{\circ} \mathrm{C}$, the supernatant was collected for western blot analysis. The protein concentration were determined using the Bradford protein assay kit (Bio-Rad Laboratories, Hercules, CA, USA). Equal amounts of protein were loaded onto a 10\% SDS-PAGE gel and then transferred onto nitrocellulose membranes (Pall Life Sciences-Pall Corp., Port Washington, NY, USA) using a wet transmembrane device (Amersham Pharmacia Biotech, Piscataway, NJ, USA). The membranes were blocked with $5 \%$ non-fat milk at room temperature for $1 \mathrm{~h}$, probed overnight with primary antibodies followed by incubation with the appropriate HRP-conjugated secondary antibody for $2 \mathrm{~h}$ at room temperature. ECL reagent (Santa Cruz Biotechnology) was used to develop the blots. All values were normalized to those of $\beta$-actin.

Immunofluorescence staining. The cfells were grown on glass coverslips and exposed to various concentrations of the drugs for the indicated periods of time. The cells were fixed with methanol for $10 \mathrm{~min}$ at $-20^{\circ} \mathrm{C}$ and permeabilized with $0.5 \%$ Triton $\mathrm{X}-100$ in PBS for $5 \mathrm{~min}$ at room temperature, and were then blocked with goat serum for $30 \mathrm{~min}$ at room temperature, incubated with primary antibody to FoxO3a (1:100; Santa Cruz Biotechnology; ) diluted in PBS overnight, and then incubated with fluorescent secondary antibody for $30 \mathrm{~min}$ at room temperature. The nucleus was counterstained with DAPI $(0.5 \mu \mathrm{g} / \mathrm{ml})$ for $10 \mathrm{~min}$ in dark. 
The coverslips with cells were examined and photographed under a fluorescence microscope (Axio Observer Inverted Microscope; Carl Zeiss, Oberkochen, Germany).

Transfection with siRNA. The HeLa cells were transfected with siRNA specific to FoxO3a (5'-ACUCCGGGUCCAGCU CCAC-3') (synthesized by Shanghai GenePharma Co., Ltd., Shanghai, China) using Lipofectamine 2000 (Invitrogen, Carlsbad,CA,USA) according to the manufacturer's instructions. A control non-specific siRNA (5'-UUCUCCGAACGUGUCA CGUTT-3') was used in parallel experiments as a negative control.

Animal studies. Nude mice were obtained from the Animal Institute of Xi'an Jiaotong University, China (XJTU). In total, 12 female 6- or 7-week old nude mice were raised in autoclave cages and supplied with unlimited water and $5 \%$ fatty food. Room temperature and humidity were maintained at $26-28^{\circ} \mathrm{C}$ and $40-60 \%$, respectively. All the animal-related procedures were approved by the Ethics Committee of the First Affiliated Hospital, and were adherent to the institutional guidelines and ethical standards. Suspensions of $1 \times 10^{6} \mathrm{HeLa}$ cells were injected subcutaneously into the flanks of nude mice. When the tumor volume was $\geq 0.1 \mathrm{~cm}^{3}$, the mice were treated intraperitoneally with butein $(2 \mathrm{mg} / \mathrm{kg} / 2$ days, $\mathrm{n}=4)$ or butein $(2 \mathrm{mg} / \mathrm{kg}$ every 2 days, $\mathrm{n}=4)+$ cisplatin $(2 \mathrm{mg} / \mathrm{kg}$ every 2 days, $\mathrm{n}=4$ ) for 3 weeks. Body weight and clinical symptoms of the mice were determined every other day. Tumor volume was calculated according to the formula: $\mathrm{V}=0.5236 \times\left(\mathrm{L} \mathrm{x} \mathrm{W}^{2}\right)$, where $\mathrm{V}$ represents the tumor volume, $\mathrm{L}$ represents the length and $\mathrm{W}$ represents the width. The animals were euthanized on day 22 following the therapeutic injection.

Histological examination. Tumor samples were fixed in $4 \%$ paraformaldehyde for $24 \mathrm{~h}$ and embedded in paraffin blocks. The sections were dewaxed in xylene, hydrated through an upgraded ethanol series and stained with H\&E, and for immunohistochemical analysis, the sections were then heated in $0.01 \mathrm{M}$ citrate buffer ( $\mathrm{pH}$ 6.0) in a steamer for $1.5 \mathrm{~min}$ to retrieve the antigen binding sites. The detection of antigens was carried out by incubation with primary antibody (FoxO3a, 1:200) for $2 \mathrm{~h}$ at room temperature, followed by incubation with HRP-labeled secondary antibody (MaxVision HRP-Polymer anti-Mouse/ Rabbit IHC kit, 1:200) at room temperature for $30 \mathrm{~min}$ and color development with DAB. The negative control specimens were incubated in PBS without the primary antibody under the same conditions. Digital images were acquired on an Olympus BH-2 microscope (Olympus, Tokyo, Japan) installed with a DeltaPix camera and software (Delta Pix, Maalov, Denmark).

Statistical analysis. Data are expressed as the means \pm SD. All statistical analyses were performed using the SPSS 18.0 statistical software package. Statistical differences were determined by the Student's t-test. Differences were considered statistically significant at $\mathrm{P}<0.05$ and highly significant at $\mathrm{P}<0.01$ for all comparisons.

\section{Results}

Butein synergistically enhances the cell growth inhibitory and apoptosis-inducing effects of cisplatin. To investigate the effectiveness of combined treatment with butein and cisplatin, the HeLa cells were treated with various concentrations of butein $(10,20$ and $40 \mu \mathrm{m})$ and/or cisplatin $(10,20$ and $30 \mu \mathrm{m})$ for different periods of time, and cytotoxicity was evaluated by MTT assay and the interaction index. Treatment with both butein and cisplatin alone inhibited cell growth in a dose- and time-dependent manner (Fig. 1A). Combined treatment with $20 \mu \mathrm{m}$ butein and $20 \mu \mathrm{m}$ cisplatin for $48 \mathrm{~h}$ induced a marked synergistic cytotoxic effect (q-values were 1.17; Fig. 1B). The apoptosis of the HeLa cells induced by butein in combination with cisplatin was further investigated by Annexin V/PI staining. As shown in Fig. 1C, treatment with butein or cisplatin alone slightly increased the percentage of apoptotic cells compared to the untreated controls, while the combinatino of both drugs significantly enhanced apoptosis. These results were in concordance with those of MTT assay, illustrating the synergistic effects of butein and cisplatin.

Co-treatment with butein and cisplatin increases $G 1$ phase arrest. A previous study demonstrated that butein induced G2/M arrest (14). Thus, in this study, we investigated whether the synergistic effects of butein and cisplatin are due to $\mathrm{G} 2 / \mathrm{M}$ cell cycle arrest, as induced by butein. In contrast to our expectations, co-treatment with butein and cisplatin induced G1 phase arrest, which differed from the effects on the cell cycle induced by treatment with butein or cisplatin alone (Fig. 1D). Furthermore, the sub-G0 DNA content was taken as a measure of the apoptotic cell population. We observed an enhancement in apoptosis in the cells treated with both butein and cisplatin compared to those treated with cisplatin alone, which is in agreement with our above-mentioned findings.

The AKT, ERK and p38 MAPK pathways are involved in the synergistic effects of butein and cisplatin. The role of the MAPK and AKT pathways in resistance to cisplatin has been previously reported (4). Since butein has been shown to exert its anticancer effects by inhibiting the AKT and MAPK signaling pathways $(11,12)$, in this study, we investigated whether these signaling pathways are associated with the enhanced growth inhibitory effects on HeLa cells following combined treatment with butein and cisplatin. As shown in Fig. 2A, butein significantly inhibited the phosphorylation of ERK and p38 induced by cisplatin, but had obvious effect on JNK expression (data not shown). Moreover, treatment with either cisplatin or butein alone or in combination inhibited AKT activation; however, p-AKT was inhibited to a greater extent following treatment with both drugs than with cisplatin alone.

Next, ERK, p38 and AKT signaling inhibitors were used to examine the functional specificity of these signaling pathways in the synergistic effects of butein and cisplatin (Fig. 2B). The HeLa cells were pre-treated with U0126 $(10 \mu \mathrm{m}) / \mathrm{SB} 203580(20 \mu \mathrm{m}) / \mathrm{LY} 294002(20 \mu \mathrm{m})$ for $8 \mathrm{~h}$ and then treated with cisplatin and/or butein for $48 \mathrm{~h}$. A significant enhancement in the cytotoxic effects of cisplatin was observed following treatment with the AKT inhibitor (LY294002), even though these cytotoxic effects (with AKT inhibitor plus cisplatin) were not as prominent as those observed following treatment with both cisplatin and butein, demonstrating that the inhibition of AKT, to a certain extent, plays a role in the synergistic effects of these drugs. Notably, a slight increase in 

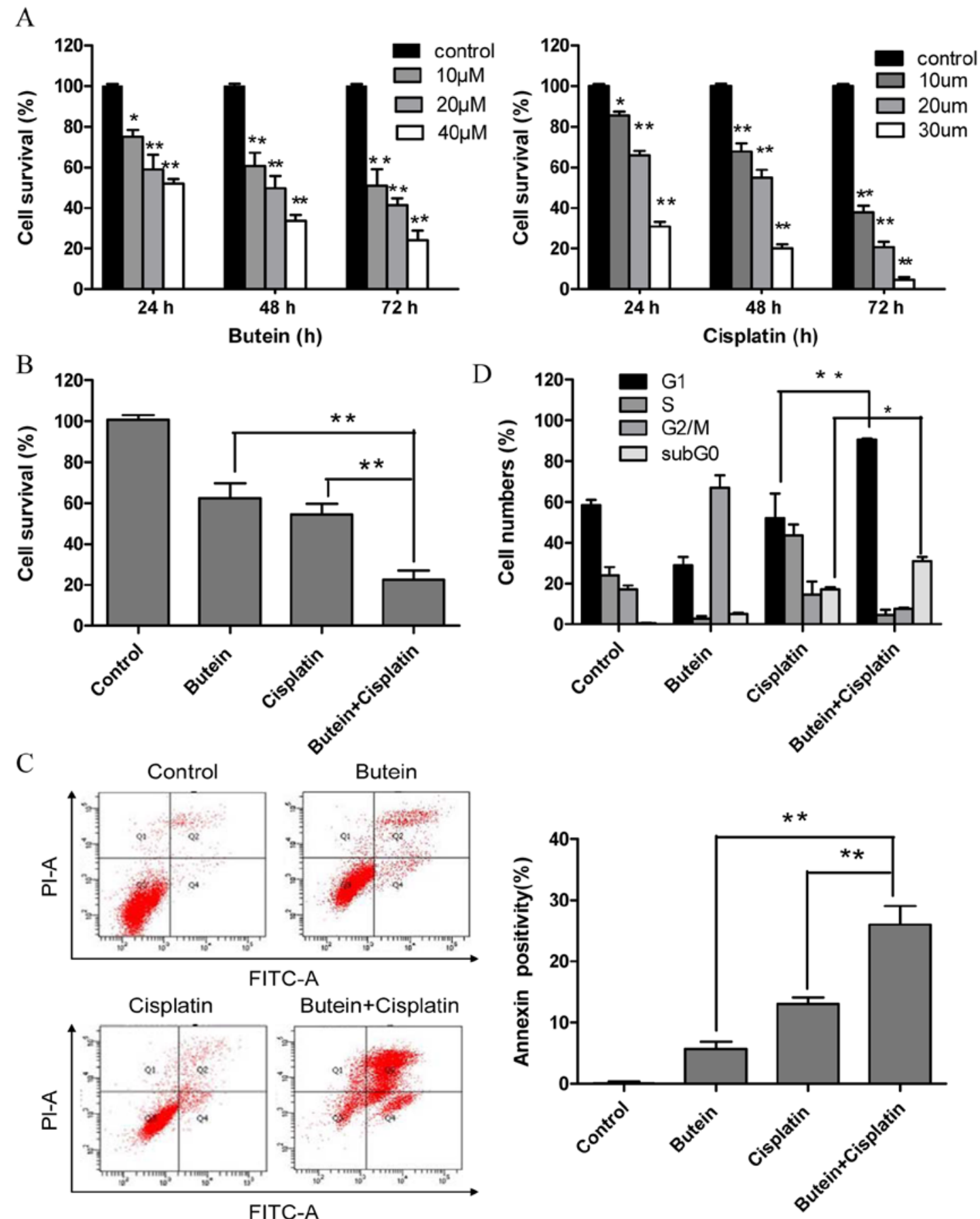

Figure 1. Effects of butein and/or cisplatin on cell proliferation, apoptosis and the cell cycle. (A) Both buein and cisplatin inhibited HeLa cell growth in a time- and dose-dependent manner. HeLa cells were treated with various concentrations of butein or cisplatin for the indicated periods of time; cell viability was determined by MTT assay. The data represent the means \pm standard deviation (SD) from 3 independent experiments $\left({ }^{*} \mathrm{P}<0.05 ;{ }^{* *} \mathrm{P}<0.01 \mathrm{vs}\right.$. control). HeLa cells were treated with butein $(20 \mu \mathrm{M})$ and/or cisplatin $(20 \mu \mathrm{M})$ for $48 \mathrm{~h}$. (B) Cell viability was assessed by MTT assay. Butein synergistically enhanced the growth inhibitory effects induced by ciaplatin on HeLa cells. (C) Apoptosis was measured by flow cytometry PI/Annexin V staining. The percentages of apoptotic cells are indicated. Butein increased the apoptosis induced by cisplatin. (D) Cell cycle progresion was measured and the apoptotic sub G0/G1 population was analyzed by flow cytometry. Butein and cisplatin in combination led to G1 phase arrest, and the quantification of cells is shown. Data are representative of at least 3 independent experiments. Data are presented as the means \pm SD from 3 independent experiments. ${ }^{*} \mathrm{P}<0.05,{ }^{* * *} \mathrm{P}<0.01$, as shown by the Student's t-test.

the cytotoxicity induced by cisplatin was observed when the cells were co-treated with the ERK (U0126) or p38 MAPK (SB203580) inhibitor as well as cisplatin; however, enhanced cytotoxic effects were observed following treatment with the ERK or p38 MAPK inhibitor in combination with butein and cisplatin. These findings were further supported by the results of apoptosis assay (Fig. 2C). Furthermore, we observed that the inhibition of AKT (with the inhibitor LY294002) decreased the phosphorylation of ERK and p38 (Fig. 3A). In addition, the inhibition of ERK by U0126 increased the phosphorylation of $\mathrm{p} 38$, and the inhibition of $\mathrm{p} 38$ by SB203580 activated ERK1/2 (with cisplatin treatment); however, the inhibition of both ERK and p38 had no obvious effect on p-AKT (Fig. 3B and $\mathrm{C}$ ). 
A
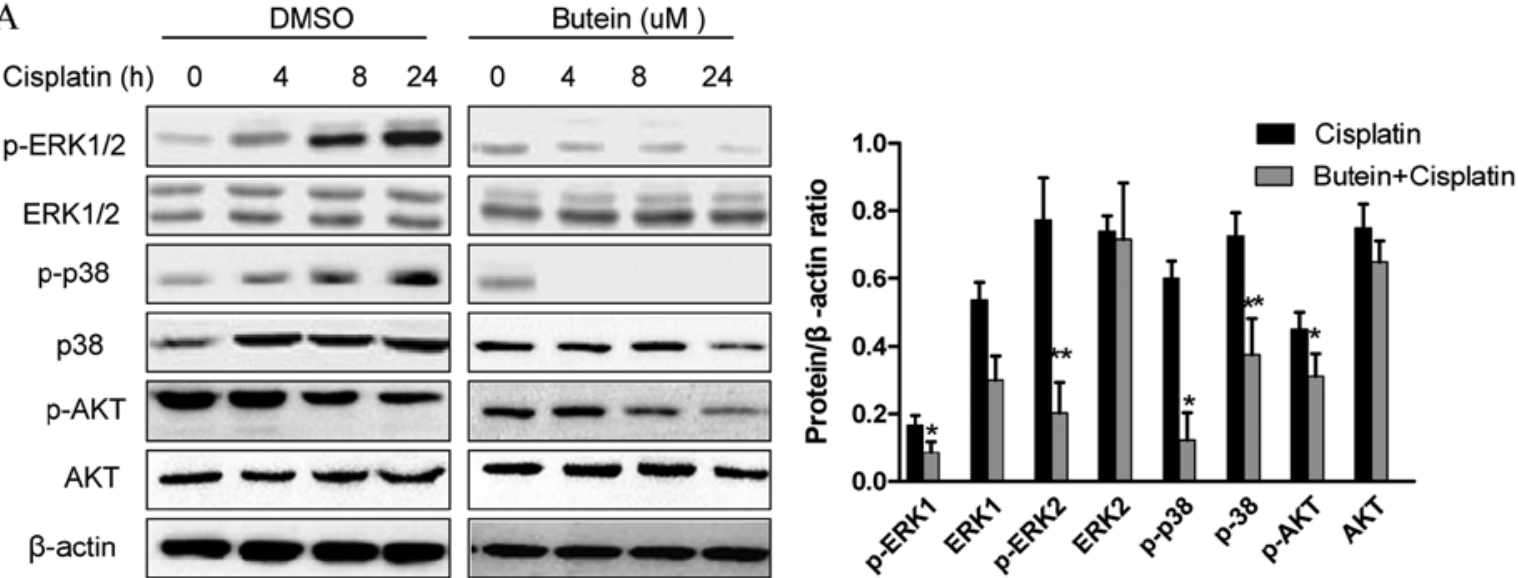

B

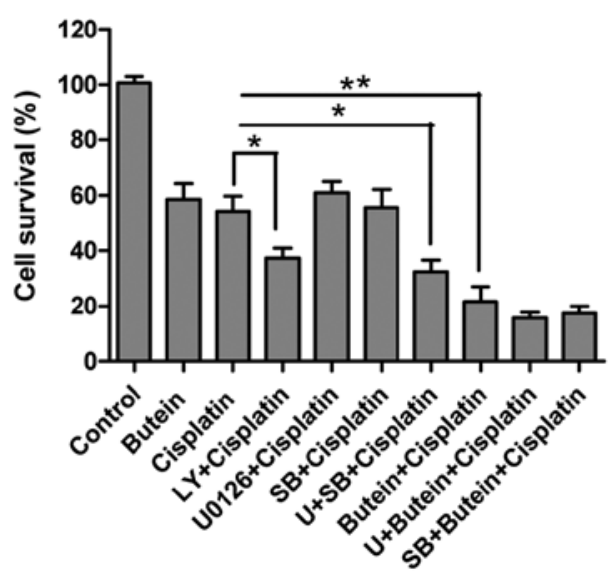

$\mathrm{C}$

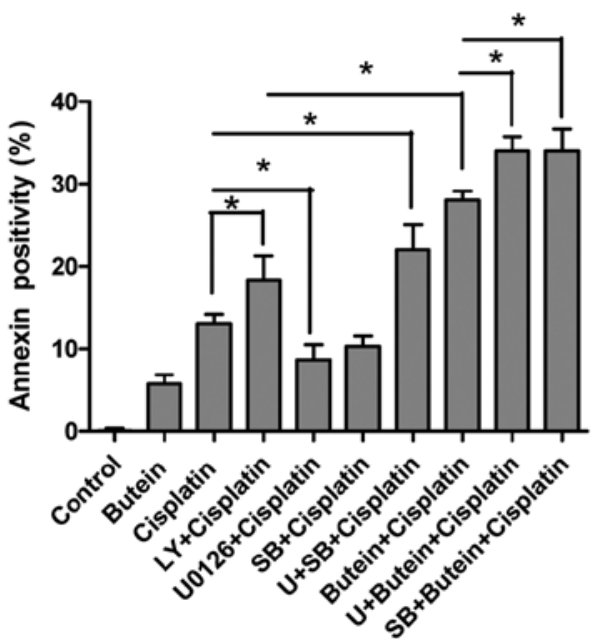

Figure 2. AKT, ERK and p38 MAPK are involved in the synergistic effects of butein and cisplatin. (A) HeLa cells were treated with butein (20 $\mu$ M) and/or cisplatin $(20 \mu \mathrm{M})$ for the indicated periods of time. Western blot analysis was performed to measure the AKT, ERK and p38 phosphorylation levels. The results revealed a decreased phosphorylation of AKT, ERK and p38 following $24 \mathrm{~h}$ of co-treatment with butein and cisplatin; $\left(\mathrm{means} \pm \mathrm{SD} ;{ }^{*} \mathrm{P}<0.05 ;{ }^{* *} \mathrm{P}<0.01, \mathrm{vs}\right.$ cisplatin alone for $24 \mathrm{~h}$ ). (B) MTT assays were performed to analyze cell viability after $48 \mathrm{~h}$ of co-treatment with U0126/SB203580/LY294002 and cisplatin/ butein. (C) Cell apoptosis were assessed by flow cytometry and PI/Annexin V staining. Data are representative of at least 3 independent experiments. Data are presented as the means \pm SD from 3 independent experiments. ${ }^{*} \mathrm{P}<0.05,{ }^{* *} \mathrm{P}<0.01$, as shown by the Student's t-test.

FoxO3a and its downstream molecules play a role in the synergistic effects of butein and cisplatin. FoxO3a, as a tumor suppressor protein, is involved in the resistance to cisplatin (15); FoxO3a can be phosphorylated and in turn degraded by AKT and ERK $(16,17)$. Moreover, a recent study revealed that the inhibition of $\mathrm{p} 38 \alpha$ in combination with chemotherapeutic agents promoted the activation of FoxO3a (18). Based on the above observations, we hypothesized that FoxO3a may be assoicated with the synergistic effects of butein and cisplatin. To examine this hypothesis, the subcellular localization and expression of FoxO3a were determined by immunofluorescence staining and western blot analysis. The results revealed that treatment of the cells with both agents led to an increased expression level of FoxO3a and to its translocation from the cytoplasm to the nucleus compared to the controls and the cells treated with cisplatin alone (Fig. 4A and B).

In order to further confirm the role of FoxO3a in the synergistic effects of cisplatin and butein, the HeLa cells were incubated for $48 \mathrm{~h}$ with siRNA targeting FoxO3a and then treated with butein and cisplatin for $48 \mathrm{~h}$. The RNAi-mediated downregulation of FoxO3a significantly decreased the apoptosis induced by combined treatment with butein and cisplatin in the HeLa cells (Fig. 4C and D). These data provide evidence that FoxO3a plays a role in the synergistic apoptotic effects induced by butein and cisplatin.

Previous studies have demonstrated that FoxO3a functions as a tumor suppressor by regulating the expression of genes involved in apoptosis, cell cycle arrest, oxidative stress, resistance and autophagy (15-17). Thus, we also examined the expression of several molecules involved in cell cycle arrest and apoptosis, which are known as downstream targets of FoxO3a. As shown in Fig. 5, butein, in combination with cisplatin, significantly enhanced the expression of p27 and decreased that of cyclin D1 compared to treatment with cisplatin alone, but had no effect on the expression of p21 (data not shown). Bax and Bcl-2 are important members of Bcl-2 family proteins and regulate mitochondrial involvement in apoptosis $(19,20)$. Co-treatment with butein and cisplatin for $48 \mathrm{~h}$ resulted in increased expression levels of Bax, but reduced protein levels of Bcl-2 compared to treatment with cisplatin alone (Fig. 5). 
A

B
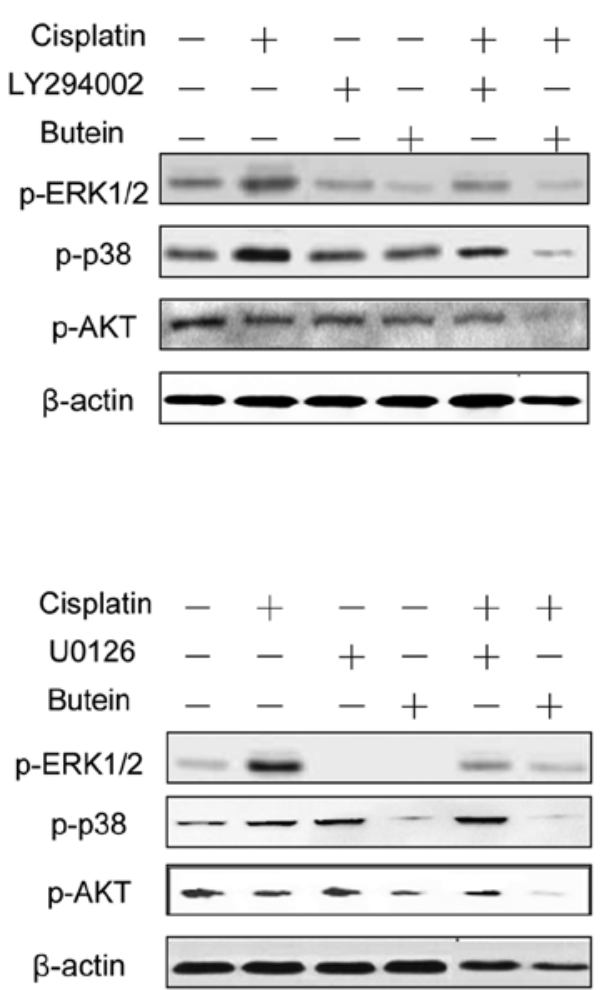

C

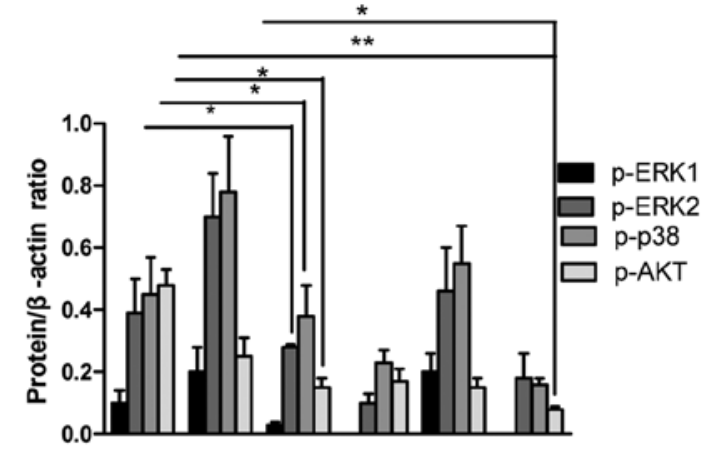

$\begin{array}{rllllll}\text { Cisplatin } & - & + & - & - & + & + \\ \text { LY294002 } & - & - & + & - & + & - \\ \text { Butein } & - & - & - & + & - & +\end{array}$

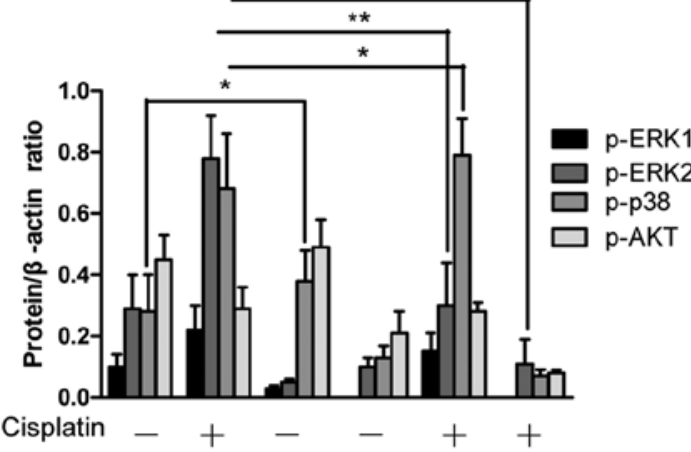

U0126 - - $6-1+$

Butein $-\quad-\quad-\quad+\quad-+$

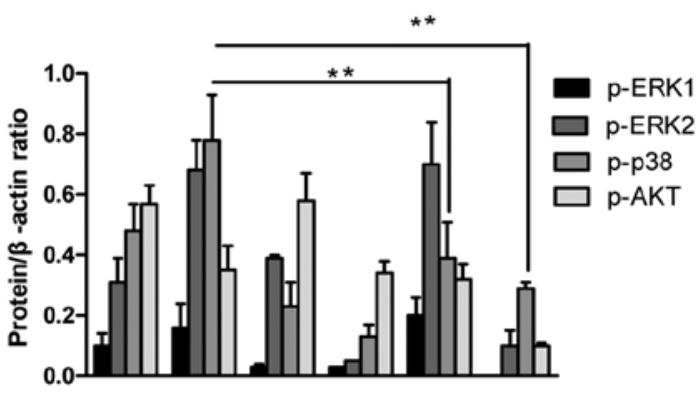

Cisplatin $-+-\quad-++$

$\mathrm{SB} 203580--+-+-$

Butein $-\quad-\quad+-+$

Figure 3. Effects of treatment with inhibitors in combination with butein/cisplatin on ERK, p38 and AKT activation. HeLa cells pre-treated with LY294002/ U0126/SB203580 were treated with cisplatin/butein for $24 \mathrm{~h}$. ERK, p38 and AKT phosphorylation was monitored by western blot analysis. Data are presented as the means $\pm \mathrm{SD}$ from 3 independent experiments. ${ }^{*} \mathrm{P}<0.05,{ }^{* *} \mathrm{P}<0.01$, as shown by the Student's t-test.

Butein in combination with cisplatin suppresses tumor growth and increases FoxO3a expression in vivo. To determine the synergistic antitumor potential of butein and cisplatin in vivo, a nude mouse tumor xenograft growth model was created. The animals were administered cisplatin and/or butein by intraperitoneal injection every 2 days when the average tumor volume was $\geq 0.1 \mathrm{~cm}^{3}$. The primary tumor sizes were monitored each week. At necropsy on day 22 following treatment, the observed inhibitory effects of cisplatin on tumor volume were significant, as compared with the controls. However, we found that combined treatment (butein + cisplatin) had the most prominent effect on tumor volume (Fig. 6A), while there was no obvious difference observed in body weight between the mice in the control group, and those treated with cisplatin alone or both drugs (Fig. 6B). We further evaluated the effects of butein on the FoxO3a expression level in the tumor tissues by immunohistochemical staining and found that the expression of FoxO3a was substantially increased in the mice treated with both agents compared with the controls and the mice treated with cisplatin alone (Fig. 6C).

\section{Discussion}

Drug resistance remains a major challenge in cancer therapy and has attracted increasing attention. In the present study, to the best of our knowledge, we investigated for the first time the 

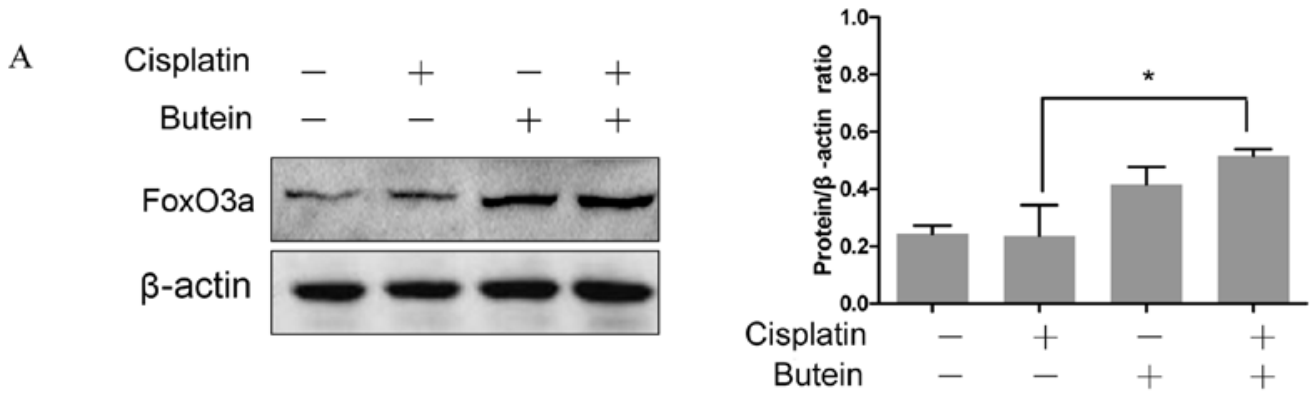

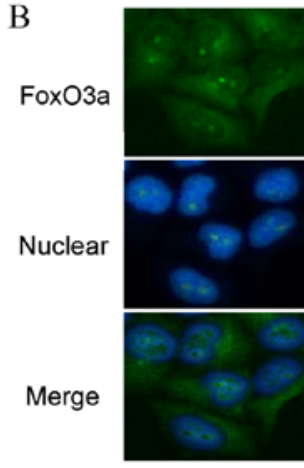

Control

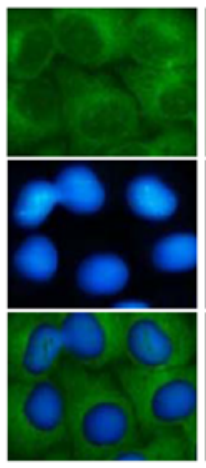

Cisplatin

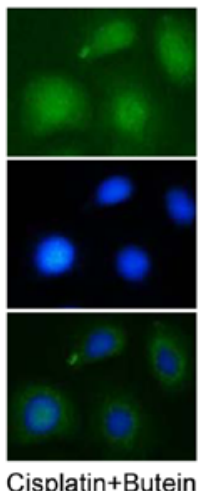

Cisplatin+Butein
D

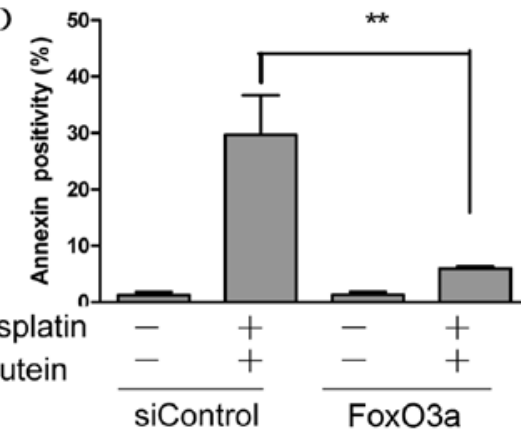

$\mathrm{C}$

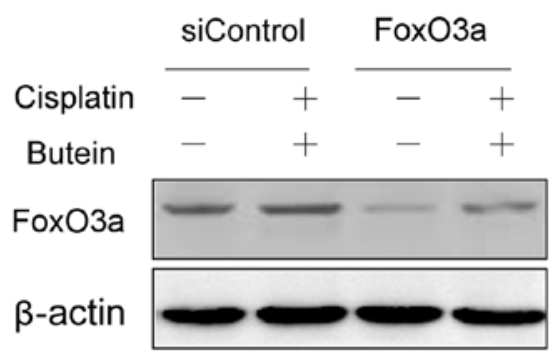

Figure 4. FoxO3a plays a role in the synergistic effects of butein and cisplatin. (A and B) Butein in combination with cisplatin results in the enhanced expression and nuclear translocation of FoxO3a. After $48 \mathrm{~h}$ of incubation of butein and/or cisplatin, the cells were examined by (A) western blot analysis and (B) immunofluorescence staining (representative fields at $\mathrm{x} 400$ magnification). (C) FoxO3a knockdown by RNAi. Cells were incubated for $48 \mathrm{~h}$ with a siRNA against FoxO3a or a control siRNA and then treated for $24 \mathrm{~h}$ with butein and cisplatin. Total cell lysates were examined by western blot analysis to confirm the downregulation of FoxO3a. (D) Cells were stained with propidium iodide (PI) and Annexin V. The percentages of apoptotic cells are indicated. Data are presented as the means \pm SD from 3 independent experiments. ${ }^{*} \mathrm{P}<0.05,{ }^{* *} \mathrm{P}<0.01$, as shown by the Student's t-test.
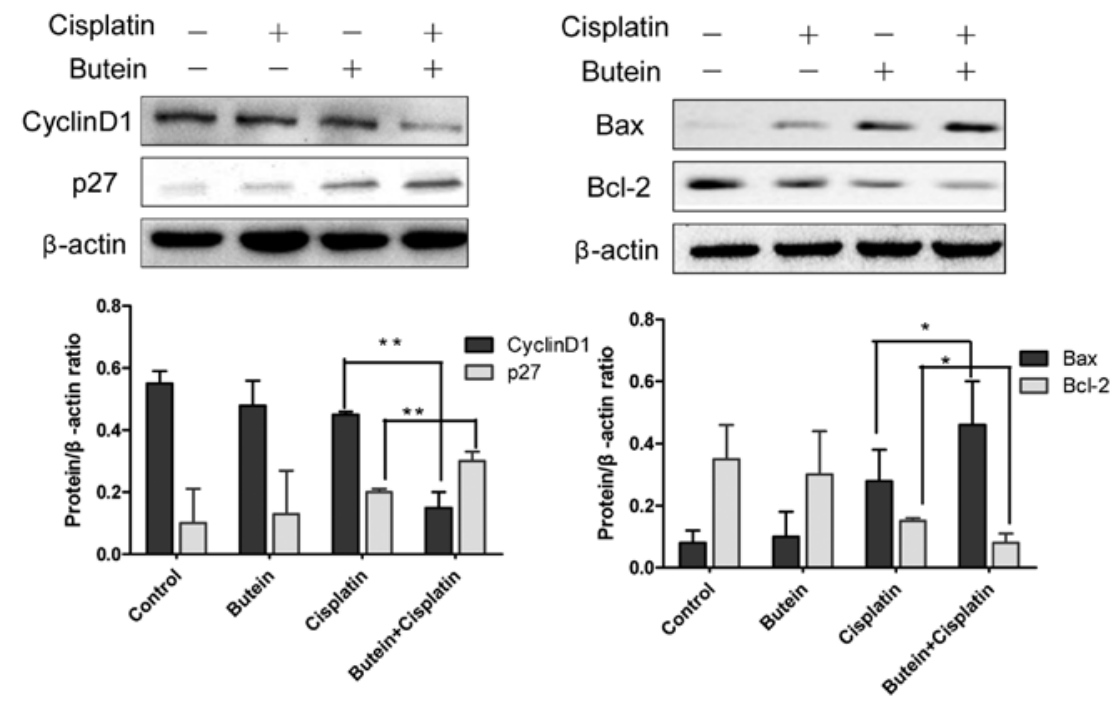

Figure 5. Effects of butein in combination with cisplatin on the expression of cyclin D1, p27, Bax and Bcl-2. Western blot analysis of cyclin D1, p27, Bax, Bcl-2 and $\beta$-actin expresion following incubation with butein and/or cisplatin for $48 \mathrm{~h}$. Data are presented as the means \pm SD from 3 independent experiments. $\mathrm{P}<0.05$, ${ }^{* *} \mathrm{P}<0.01$, as shown by the Student's t-test. 


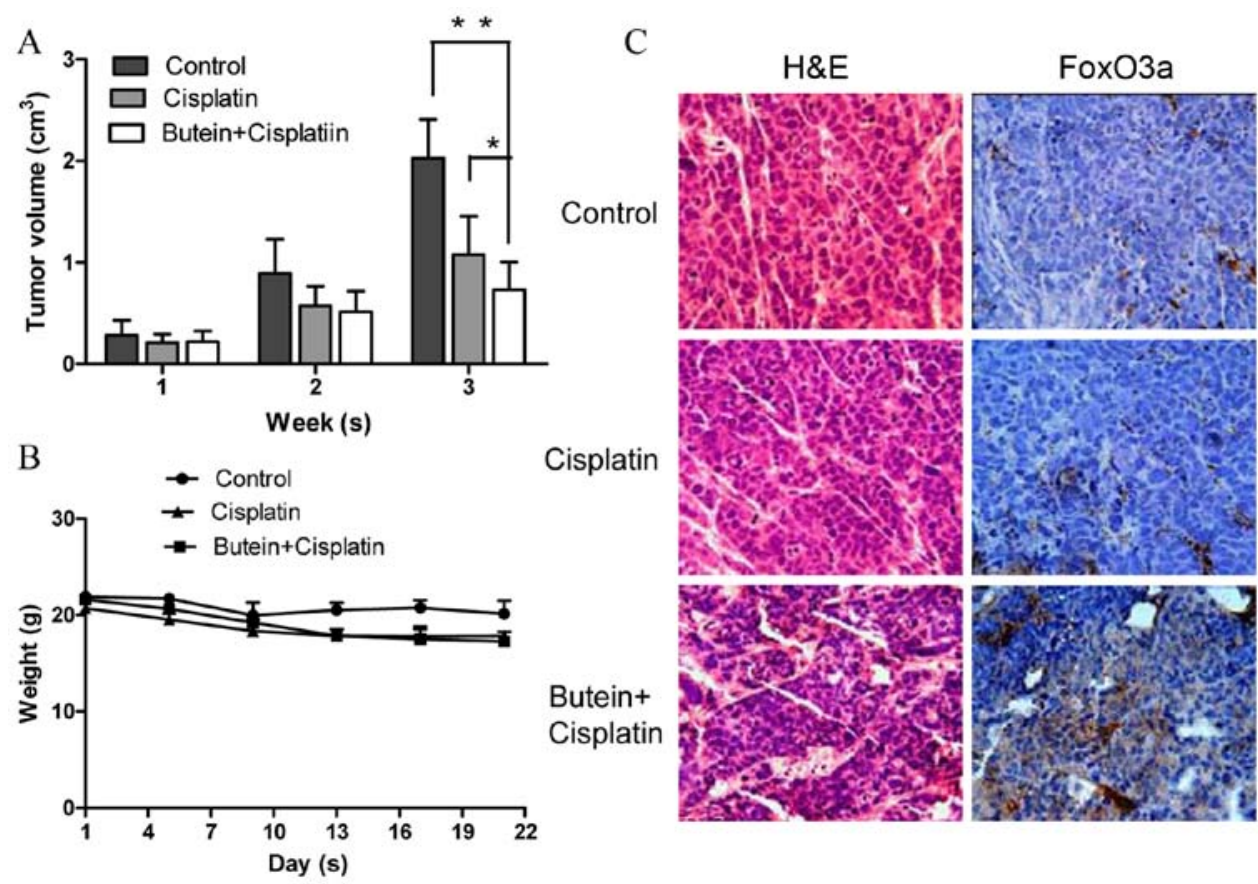

Figure 6. Butein enhances the antitumor effects of cisplatin in vivo. (A) Athymic mice bearing subcutaneous tumors were treated intraperitoneally with PBS, cisplatin or butein + cisplatin, and the tumor volume was calculated. Data represent the means $\left(\mathrm{cm}^{3}\right) \pm \mathrm{SD}\left(\mathrm{n}=4\right.$ per group). ${ }^{*} \mathrm{P}<0.05,{ }^{* *} \mathrm{P}<0.01$, as shown by the Student's t-test. (B) Body weight of nude mice with subcutaneous tumor xenografts was monitored every other day. No obvious differences in body weight were observed between the controls, or the mice treated with cisplatin or butein alone. Values are presented as the means $(g) \pm S D(n=4$ per group). (C) Immunohistochemical staining of FoxO3a in subcutaneous tumor samples revealed increased expression levels of FoxO3a in the group treated with both agents, as compared with the controls and the group treated with cisplatin alone. Images were acquired at x400 magnification.

synergistic effects of butein and cisplatin on cervical cancer cell growth inhibition and apoptosis in vitro and in vivo, and further explored the possible mechanisms responsible for their synergistic effects.

The anticancer effects of butein have been well documented in various types of cancer (6-10). Our results also indicated that butein inhibited HeLa cell proliferation in a dose- and timedependent manner. However, to the best of our knowledge, no previous studies on the possible synergistic anticancer effects of butein and cisplatin have been published to date. In the present study, we found that butein enhanced the growth inhibitory and apoptotic effects induced by cisplatin. The underlying molecular mechanisms were also explored. Although the signaling pathways activated by DNA damage are different from the types of DNA damage, the activation of these pathways has similar results, including cell cycle arrest and ultimately, either cell survival or cell death. In this study, butein induced $\mathrm{G} 2 / \mathrm{M}$ arrest, which is in accordance with the findings of a previous study (14). We speculated that the synergistic effects of butein and cisplatin may be due to G2/M cell cycle arrest. In contrast to our expectations, co-treatment with butein and cisplatin increased G1 phase arrest, suggesting that enhanced G1 phase arrest plays a role in the synergistic apoptotic effects induced by combined treatment with butein and cisplatin.

It has been demonstrated that chemoresistance is due to survival signaling pathways activated during chemotherapy $(2,21)$. In the present study, we found that butein markedly reduced the phosphorylation and activation of ERK, p38 and AKT in the presence of cisplatin, but had no obvious effect on JNK (data not shown). Therefore, it is possible that butein blocks the signaling circuit involving ERK/p38 MAPK and
AKT, acting as a chemosensitizer. The ERK pathway is widely accepted as an important survival regulator, and the chemical inhibition of the ERK pathway has been shown to sensitize cells to cisplatin $(22,23)$. However, studies have produced different data and have shown that ERK activation is required in cisplatininduced apoptosis $(24,25)$; the inhibition of ERK activation has also been shown to markedly attenuate cisplatin-induced cell death $(26,27)$. The biological effects of p38 activation are also highly conflicting. The inhibition of p38 by SB203580 has been shown to significantly block Met-induced apoptosis in A549/ CDDP cells (28), while other researchers have found that the inhibition of p38 sensitizes breast and gastric cancer cells to cisplatin-induced apoptosis $(29,30)$ and enhanced p38 activation has been associated with a poor overall survival in patients with breast cancer (31) and hepatocellular carcinoma (32). It has been reported that $\mathrm{p} 38$ enhances cancer cell growth after the acquisition of the malignant phenotype, and acts as a tumor suppressor, mainly at the onset of cellular transformation $(33,34)$. Thus, the role of ERK and p38 appears to be dependent upon the cellular context and stimuli. The constitutive activation of AKT has also been implicated in chemoresistance (35), while the inactivation of AKT signaling by chemicals sensitizes human cancer cells to cisplatin (36).

To further identify the functional specificities of these pathways in the synergistic effects of butein and cisplatin, in this study, we used AKT and ERK/p38 MAPK inhibitors. The results revealed that the AKT inhibitor enhanced the apoptotic effects of cisplatin, although the apoptotic effects induced by the AKT inhibitor in combination with cisplatin were not as prominent as those induced by combined treatment with butein and cisplatin. These findings suggest that butein exerts 
its sensitizing effects on cisplatin, to a certain extent, through the regulation of AKT, and that other signaling pathways are involved in the synergistic effects. We then found the dual inhibition of ERK/p38 MAPK and ERK or p38 in combination with butein enhanced the apoptosis induced by cisplatin, indicating that the inhibition of ERK and p38 by butein also plays a role in the synergistic effects of butein and cisplatin. Of note, we found that the inhibition of ERK promoted $\mathrm{p} 38$ activation, and the slight activation of ERK was also observed upon the inhibition of p38 in combination with cisplatin treatment, although no significant difference was observed. In fact, ERK inhibition triggers p38 activation in HeLa cells (37), and p38 inhibition has also been reported to upregulate the activation of the MEK-ERK1/2 survival pathway (38). A recent study by Chiacchiera et al (38) demonstrated that the combined inhibition of $\mathrm{p} 38 \alpha$ and MEK specifically induced apoptosis through caspase- 3 in colorectal cancer cells. These data indicate that there is a crosstalk between the ERK and p38 pathways, which is crucial for the therapeutic response. In addition, we observed that the AKT inhibitor decreased the phosphorylation of ERK and p38, while the inhibition of ERK and p38 had no effects on AKT activation, indicating that AKT may be the upstream signal of ERK and p38.

FoxO3a has been investigated as a crucial protein that is involved in the regulation of cell survival and proliferation, contributing to tumor suppression (16). The AKT and ERK-mediated phosphorylation of FoxO3a stimulate its ubiquitination, resulting in proteasomal degradation $(17,40)$. AKT directly phosphorylates FoxO3a at S253, which is a crucial residue regulating the nuclear/cytoplasmic shuttling of FoxO3a. FoxO3a localization in the cytoplasm is a key step leading to FoxO3a deactivation and degradation, and correlates with poor survival in patients with breast cancer (39). Studies have found that FoxO3a may be a key molecule of the p38 pathway and may be involved in drug resistance $(41,42)$. Since butein inhibited the AKT, ERK and p38 MAPK pathways, which are all involved in the regulation of FoxO3a, we subsequently examined whether FoxO3a is a key molecule involved in the synergistic effects of butein and cisplatin. We found that combined treatment with butein and cisplatin increased the nuclear translocation and expression of FoxO3a compared to treatment with cisplatin alone, and the downregulation of FoxO3a by RNAi significantly inhibited the synergistic effects of butein and cisplatin in HeLa cells, suggesting that butein exerts its chemosensitizing effects, in part through FoxO3a activation. Our in vivo findings revealed that butein and cisplatin exerted similar inhibitory effects on tumor growth, by increasing the FoxO3a protein level.

Activated FoxO3a is able to bind to promoters and induces the transcription of target genes, which include p21, p27 and cyclin D1 for cell cycle arrest, and Bim, Bcl-2 and Bax for cell apoptosis (43-45). Alterations in cell cycle progression in various tumors are often due to mutations or the overexpression of genes. As an inhibitor of cyclin E-Cdk2, p27 plays a pivotal role in controlling cell G1-S phase transition during development and tumorigenesis. In addition, cyclin D1 mediates the G1/S transition by binding to Cdk4 and also by sequestering a Cdk inhibitor of p21 and p27 (46). The present study demonstrated that the upregulation of p27 and the downregulation of cyclin D1 expression was induced by combined treatment with butein and cisplatin compared to treatmetn with cisplatin alone, which coincided with G1 phase arrest. This suggests that cyclin D1 and p27, two important regulators of the cell cycle, are intracellular targets of the butein-mediated anti-proliferative effects on HeLa cells through FoxO3a activation. The Bcl-2 family of proteins are important regulators of apoptosis $(47,48)$. In this study, the apoptosis induced by butein in HeLa cells was associated with the downregulation of anti-apoptotic $\mathrm{Bcl}-2$ expression and an increased Bax expression.

Overall, the findings of this study reveal a new function of butein that enhances the sensitization of cervical cancer cells to cisplatin in vitro and in vivo, which may be related to the AKT and ERK/p38 MAPK pathways, at least to a certain extent, through the regulation of FoxO3a. These data shed some light on the synergistic antitumor effects of butein and cisplatin and verify the potential clinical use of butein.

\section{Acknowledgements}

The present study was supported by grants from the National Natural Science Foundation of China (no. 81202055); the Key Sci-tech Project of Shaanxi Province (no. 2012SF2-03) and the Sci-tech Project of Xi'an City (no. SF1204(56).

\section{References}

1. Basu A and Krishnamurthy S: Cellular responses to cisplatininduced DNA damage. J Nuleic Acids 2010: Article ID 201367, 2010.

2. Brozovic A and Osmak M: Activation of mitogen-activated protein kinases by cisplatin and their role in cisplatin-resistance. Cancer Lett 251: 1-16, 2007.

3. Wang Z, Hou J, Lu L, Qi Z, Sun J, Gao W, Meng J, Wang Y, Sun H, Gu H, et al: Small ribosomal protein subunit S7 suppresses ovarian tumorigenesis through regulation of the PI3K/AKT and MAPK pathways. PLoS One 8: e79117, 2013.

4. Bava SV, Puliappadamba VT, Deepti A, Nair A, Karunagaran D and Anto RJ: Sensitization of taxol-induced apoptosis by curcumin involves down-regulation of nuclear factor-kappaB and the serine/threonine kinase Akt and is independent of tubulin polymerization. J Biol Chem 280: 6301-6308, 2005.

5. Kang DG, Lee AS, Mun YJ, Woo WH, Kim YC, Sohn EJ, Moon MK and Lee HS: Butein ameliorates renal concentrating ability in cisplatin-induced acute renal failure in rats. Biol Pharm Bull 27: 366-370, 2004.

6. Wang Y, Chan FL, Chen S and Leung LK: The plant polyphenol butein inhibits testosterone-induced proliferation in breast cancer cells expressing aromatase. Life Sci 77: 39-51, 2005.

7. Cho SG, Woo SM and Ko SG: Butein suppresses breast cancer growth by reducing a production of intracellular reactive oxygen species. J Exp Clin Cancer Res 33: 51, 2014.

8. Yit CC and Das NP: Cytotoxic effect of butein on human colon adenocarcinoma cell proliferation. Cancer Lett 82: 65-72, 1994.

9. Ma CY, Ji WT, Chueh FS, Yang JS, Chen PY, Yu CC and Chung JG: Butein inhibits the migration and invasion of SK-HEP-1 human hepatocarcinoma cells through suppressing the ERK, JNK, p38, and uPA signaling multiple pathways. J Agric Food Chem 59: 9032-9038, 2011.

10. Zhang L, Chen W and Li X: A novel anticancer effect of butein: Inhibition of invasion through the ERK1/2 and NF-kappa B signaling pathways in bladder cancer cells. FEBS Lett 582: 1821-1828, 2008.

11. Lau GT, Huang H, Lin SM and Leung LK: Butein downregulates phorbol 12-myristate 13-acetate-induced COX-2 transcriptional activity in cancerous and non-cancerous breast cells. Eur J Pharmacol 648: 24-30, 2010.

12. Khan N, Adhami VM, Afaq F and Mukhtar H: Butein induces apoptosis and inhibits prostate tumor growth in vitro and in vivo. Antioxid Redox Signal 16: 1195-1204, 2012. 
13. Jin ZJ: About the evaluation of drug combination. Acta Pharmacol Sin 25: 146-147, 2004

14. Moon DO, Kim MO, Choi YH, Hyun JW, Chang WY and Kim GY: Butein induces $\mathrm{G}(2) / \mathrm{M}$ phase arrest and apoptosis in human hepatoma cancer cells through ROS generation. Cancer Lett 288: 204-213, 2010.

15. Shiota M, Yokomizo A, Kashiwagi E, Tada Y, Inokuchi J, Tatsugami K, Kuroiwa K, Uchiumi T, Seki N and Naito S: Foxo3a expression and acetylation regulate cancer cell growth and sensitivity to cisplatin. Cancer Sci 101: 1177-1185, 2010.

16. Khatri S, Yepiskoposyan H, Gallo CA, Tandon P and Plas DR: FOXO3a regulates glycolysis via transcriptional control of tumor suppressor TSC1. J Biol Chem 285: 15960-15965, 2010.

17. Yang W, Dolloff NG and El-Deiry WS: ERK and MDM2 prey on FOXO3a. Nat Cell Biol 10: 125-126, 2008.

18. Germani A, Matrone A, Grossi V, Peserico A, Sanese P, Liuzzi M, Palermo R, Murzilli S, Campese AF, Ingravallo G, et al: Targeted therapy against chemoresistant colorectal cancers: Inhibition of $\mathrm{p} 38 \alpha$ modulates the effect of cisplatin in vitro and in vivo through the tumor suppressor FoxO3A. Cancer Lett 344: $110-118,2014$.

19. Quast SA, Berger A, Plötz M and Eberle J: Sensitization of melanoma cells for TRAIL-induced apoptosis by activation of mitochondrialpathways via Bax. Eur J Cell Biol 93: 42-48, 2014.

20. Kim MJ, Yun HS, Hong EH, Lee SJ, Baek JH, Lee CW, Yim JH, Kim JS, Park JK, Um HD and Hwang SG: Depletion of end-binding protein 1 (EB1) promotes apoptosis of human non-small-cell lung cancer cells via reactive oxygen species and Bax-mediated mitochondrial dysfunction. Cancer Lett 339: 15-24, 2013.

21. Hsu HH, Cheng LH, Ho TJ, Kuo WW, Lin YM, Chen MC, Lee NH, Tsai FJ, Tsai KH and Huang CY: Apicidin-resistant HA22T hepatocellular carcinoma cells massively promote pro-survival capability via IGF-IR/PI3K/Akt signaling pathway activation. Tumour Biol 35: 303-313, 2014.

22. Persons DL, Yazlovitskaya EM, Cui W and Pelling JC: Cisplatininduced activation of mitogen-activated protein kinases in ovarian carcinoma cells: Inhibition of extracellular signalregulated kinase activity increases sensitivity to cisplatin. Clin Cancer Res 5: 1007-1014, 1999.

23. Wang J,Zhou JY and Wu GS: Bim protein degradation contributes to cisplatin resistance. J Biol Chem 286: 22384-22392, 2011.

24. Wang X, Martindale JL and Holbrook NJ: Requirement for ERK activation in cisplatin-induced apoptosis. J Biol Chem 275: 39435-39443, 2000.

25. Wang X, Govind S, Sajankila SP, Mi L, Roy R and Chung FL: Phenethyl isothiocyanate sensitizes human cervical cancer cells to apoptosis induced by cisplatin. Mol Nutr Food Res 55: 1572-1581, 2011.

26. Sheridan C, Brumatti G, Elgendy M, Brunet M and Martin SJ: An ERK-dependent pathway to Noxa expression regulates apoptosis by platinum-based chemotherapeutic drugs. Oncogene 29: 6428-6441, 2010.

27. Guégan JP, Ezan F, Théret N, Langouët S and Baffet G: MAPK signaling in cisplatin-induced death: Predominant role of ERK1 over ERK2 in human hepatocellular carcinoma cells. Carcinogenesis 34: 38-47, 2013.

28. Wang Y, Lin B, Wu J, Zhang $\mathrm{H}$ and $\mathrm{Wu}$ B: Metformin inhibits the proliferation of A549/CDDP cells by activating p38 mitogenactivated protein kinase. Oncol Lett 8: 1269-1274, 2014

29. Pereira L, Igea A, Canovas B, Dolado I and Nebreda AR: Inhibition of p38 MAPK sensitizes tumour cells to cisplatininduced apoptosis mediated by reactive oxygen species and JNK. EMBO Mol Med 5: 1759-1774, 2013.

30. Feng R, Zhai WL, Yang HY, Jin H and Zhang QX: Induction of ER stress protects gastric cancer cells against apoptosis induced by cisplatin and doxorubicin through activation of p38 MAPK. Biochem Biophys Res Commun 406: 299-304, 2011.

31. Esteva FJ, Sahin AA, Smith TL, Yang Y, Pusztai L, Nahta R, Buchholz TA, Buzdar AU, Hortobagyi GN and Bacus SS: Prognostic significance of phosphorylated P38 mitogen-activated protein kinase and HER-2 expression in lymph node-positive breast carcinoma. Cancer 100: 499-506, 2004.
32. Wang SN, Lee KT, Tsai CJ, Chen YJ and Yeh YT: Phosphorylated p38 and JNK MAPK proteins in hepatocellular carcinoma. Eur J Clin Invest 42: 1295-1301, 2012.

33. Dolado I, Swat A, Ajenjo N, De Vita G, Cuadrado A and Nebreda AR: p38alpha MAP kinase as a sensor of reactive oxygen species in tumorigenesis. Cancer Cell 11: 191-205, 2007.

34. Voisset E, Oeztuerk-Winder F, Ruiz EJ and Ventura JJ: p38a negatively regulates survival and malignant selection of transformed bronchioalveolar stem cells. PLoS One 8: e78911, 2013.

35. Piaggi S, Raggi C, Corti A, Pitzalis E, Mascherpa MC, Saviozzi M, Pompella A and Casini AF: Glutathione transferase omega 1-1 (GSTO1-1) plays an anti-apoptotic role in cell resistance to cisplatin toxicity. Carcinogenesis 31: 804-811, 2010.

36. Ishitsuka A, Fujine E, Mizutani Y, Tawada C, Kanoh H, Banno Y and Seishima M: FTY720 and cisplatin synergistically induce the death of cisplatin-resistant melanoma cells through the downregulation of the PI3K pathway and the decrease in epidermal growth factor receptor expression. Int J Mol Med 34: 1169-1174, 2014.

37. Berra E, Diaz-Meco MT and Moscat J: The activation of p38 and apoptosis by the inhibition of Erk is antagonized by the phosphoinositide 3-kinase/Akt pathway. J Biol Chem 273: 10792-10797, 1998.

38. Chiacchiera F, Grossi V, Cappellari M, Peserico A, Simonatto M, Germani A, Russo S, Moyer MP, Resta N, Murzilli S, et al: Blocking p38/ERK crosstalk affects colorectal cancer growth by inducing apoptosis in vitro and in preclinical mouse models. Cancer Lett 324: 98-108, 2012.

39. Hu MC, Lee DF, Xia W, Golfman LS, Ou-Yang F, Yang JY, Zou Y, Bao S, Hanada N, Saso H, et al: IkappaB kinase promotes tumorigenesis through inhibition of forkhead FOXO3a. Cell 117: 225-237, 2004

40. Yang JY, Zong CS, Xia W, Yamaguchi H, Ding Q, Xie X, Lang JY, Lai CC, Chang CJ, Huang WC, et al: ERK promotes tumorigenesis by inhibiting FOXO3a via MDM2-mediated degradation. Nat Cell Biol 10: 138-148, 2008.

41. Chiacchiera F, Matrone A, Ferrari E, Ingravallo G, Lo Sasso G, Murzilli S, Petruzzelli M, Salvatore L, Moschetta A and Simone C: p38alpha blockade inhibits colorectal cancer growth in vivo by inducing a switch from HIFlalpha- to FoxO-dependent transcription. Cell Death Differ 16: 1203-1214, 2009.

42. Chiacchiera F and Simone C: Inhibition of p38alpha unveils an AMPK-FoxO3A axis linking autophagy to cancer-specific metabolism. Autophagy 5: 1030-1033, 2009.

43. Zhang X, Tang N, Hadden TJ and Rishi AK: Akt, FoxO and regulation of apoptosis. Biochim Biophys Acta 1813: 1978-1986, 2011.

44. Ho KK, Myatt SS and Lam EW: Many forks in the path: Cycling with FoxO. Oncogene 27: 2300-2311, 2008.

45. Schmidt M, Fernandez de Mattos S, van der Horst A, Klompmaker R, Kops GJ, Lam EW, Burgering BM and Medema RH: Cell cycle inhibition by FoxO forkhead transcription factors involves downregulation of cyclin D. Mol Cell Biol 22: 7842-7852, 2002.

46. Massagué J: G1 cell-cycle control and cancer. Nature 432: 298-306, 2004

47. Lee MT, Ho SM, Tarapore P, Chung I and Leung YK: Estrogen receptor $\beta$ isoform 5 confers sensitivity of breast cancer cell lines to chemotherapeutic agent-induced apoptosis through interaction with Bcl2L12. Neoplasia 15: 1262-1271, 2013.

48. Kiyoshima T1, Yoshida H, Wada H, Nagata K, Fujiwara H, Kihara M, Hasegawa K, Someya H and Sakai H: Chemoresistance to concanamycin A1 in human oral squamous cell carcinoma is attenuated by an HDAC inhibitor partly via suppression of Bcl-2 expression. PLoS One 8: e80998, 2013. 